\title{
Central nervous system vasculitis after chickenpox-cause or coincidence?
}

\author{
Avinoam Shuper, Eileen P G Vining, John M Freeman
}

\begin{abstract}
A 7.5 year old boy, known to have a seizure disorder, presented with an infarct in the left middle cerebral artery territory, 10 weeks after severe chickenpox. Immunofluorescent antibody titre to the varicella zoster virus in the cerebrospinal fluid was 1:32. Cerebral angiography showed evidence of focal vasculitis. He presented again seven months later with an acute exacerbation of seizures. Magnetic resonance imaging of the brain showed an old posterior extension of the infarct, but a repeated angiography demonstrated an improvement in the vasculitic process. Cerebrospinal fluid antibody titre was again 1:32. Although this may have been an unfortunate coincidence, a possible association between chickenpox and vasculitis, similar to that reported with herpes zoster, and with potentially significant clinical implications, should be considered. As a definite proof can be obtained only by a brain biopsy, however, which is generally not indicated in such cases, only additional clinical reports can lead to delineation of this association as a definite entity.
\end{abstract}

Nervous system complications of varicella are not rare in general paediatric practice. A diversity of neurological conditions, including meningoencephalitis, acute cerebellar ataxia, and the Guillain-Barré and Reye's syndromes have been reported. ${ }^{1}$ The varicella zoster virus may cause cerebral angiitis after herpes zoster ophthalmicus, ${ }^{2}$ and angiitis in adults with and without immune deficiencies after other herpes zoster infections not involving the trigeminal nerve. $^{3-5}$ The association of vascular brain lesions after chickenpox, although well described, ${ }^{6-9}$ is much less well recognised. We describe a child with cerebral vasculitis resulting in acute aphasia and hemiplegia 10 weeks after the onset of chickenpox. The dilemmas resulting from this possible association are discussed.

Department of Division of

Pediatric Neurology, The Johns Hopkins Medical Institution, $600 \mathrm{~N}$ Wolfe Street, Baltimore, MD 21205 ,

USA

Avinoam Shupe

Evinoam Shuper John M Freeman

Correspondence to: Dr Shuper.

Accepted 25 June 1990 mental quotient of around 70. At age 3 years he developed a mixed seizure disorder with generalised tonic-clonic seizures, partial right sided motor seizures, and drooling spells. His neurodevelopmental problems were thoroughly evaluated in another hospital, with negative results. Of note, plasma and urine amino acid pattern and magnetic resonance imaging of the brain, which were done at the age of 5.5 years, were normal. Physical and neurological examinations were unremarkable. Seizures were well controlled by the use of valproic acid, $1125 \mathrm{mg}$ daily.

Chickenpox developed 10 weeks before his hemiparesis and was described as severe and widespread, with no signs of central nervous system involvement. The lesions crusted and the illness resolved after about two weeks.

Twenty four hours before his admission he was noted to have weakness in his right hand, which progressed over hours to involve the arm, after which right sided facial weakness with drooling developed, followed by inability to speak. The next day he had a right hemiplegia and expressive and receptive aphasia.

Physical examination was remarkable for numerous generalised healing chickenpox scars. He had a profound expressive aphasia, weakness of the right lower half of his face, and motor weakness ( 3 in a scale of 0 to 5 ) on the right. The deep tendon reflexes were hyperactive and his toe was 'up going' on the same side. Sensory examination was normal. His right arm and leg were mildly tremulous.

On admission his packed cell volume fraction was 0.35 , white cell count $8.9 \times 10^{9} / 1$ with differential count fractions of 0.08 band forms, 0.3 polymorphonuclears, 0.5 lymphocytes, 0.11 monocytes, and 0.01 atypical lymphocyte. The sedimentation rate was $6 \mathrm{~mm}$ in the first hour. Concentrations of serum electrolytes, results of liver function tests and determinations of anion gap, prothrombin time, and activated partial thromboplastin time gave normal results. Blood lactate concentration was 0.6 $\mathrm{mmol} / \mathrm{l}$ (normal up to $3.0 \mathrm{mmol} / \mathrm{l}$ ) and lactate in the cerebrospinal fluid was $1.6 \mathrm{mmol} / \mathrm{l}$. Rheumatoid factor and anti-DNA antibodies were negative, antinuclear antibodies were positive at a titre of 1:40 (not significant below 1:160). A lumbar puncture showed normal cerebrospinal fluid pressure, normal concentrations of glucose and protein, and 1 mononuclear white blood cell $/ \mathrm{ml}$ and 2 red blood cells $/ \mathrm{ml}$. The antivaricella immunofluorescent antibody titre was 1:1024 in the peripheral blood and $1: 32$ in the cerebrospinal fluid.

Electrocardiography and echocardiography were normal. Electroencephalography showed a consistent focal slowing over the left central parietal region, and recurrent bursts of bilateral 
spikes, which occasionally were more apparent over the right hemisphere.

Magnetic resonance imaging of the head (fig 1) was compatible with acute left temporal and parietal lobe infarct with an abnormal signal intensity within the left basal ganglia, compatible with lacunar infarcts. Bilateral cerebral arteriography showed long discontinuous segments of smooth, concentric narrowing, and beading involving the left anterior and predominantly the middle cerebral arteries, and some involvement of the left posterior cerebral artery (fig 2). The beading angioarchitecture of

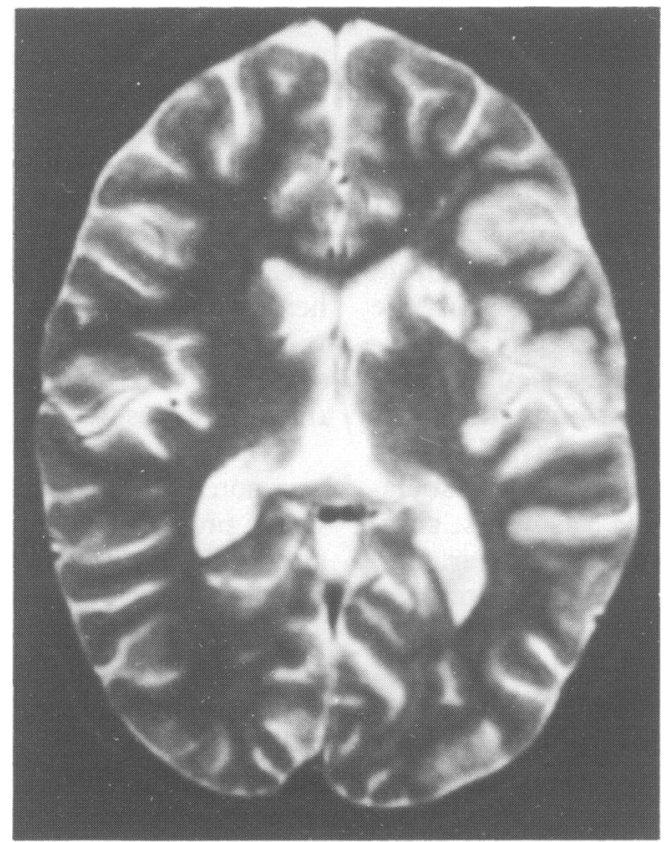

Figure 1 Magnetic resonance imaging of the brain showing the acute infarct in the left temporal and parietal lobes, with the involvement of the left basal ganglia.

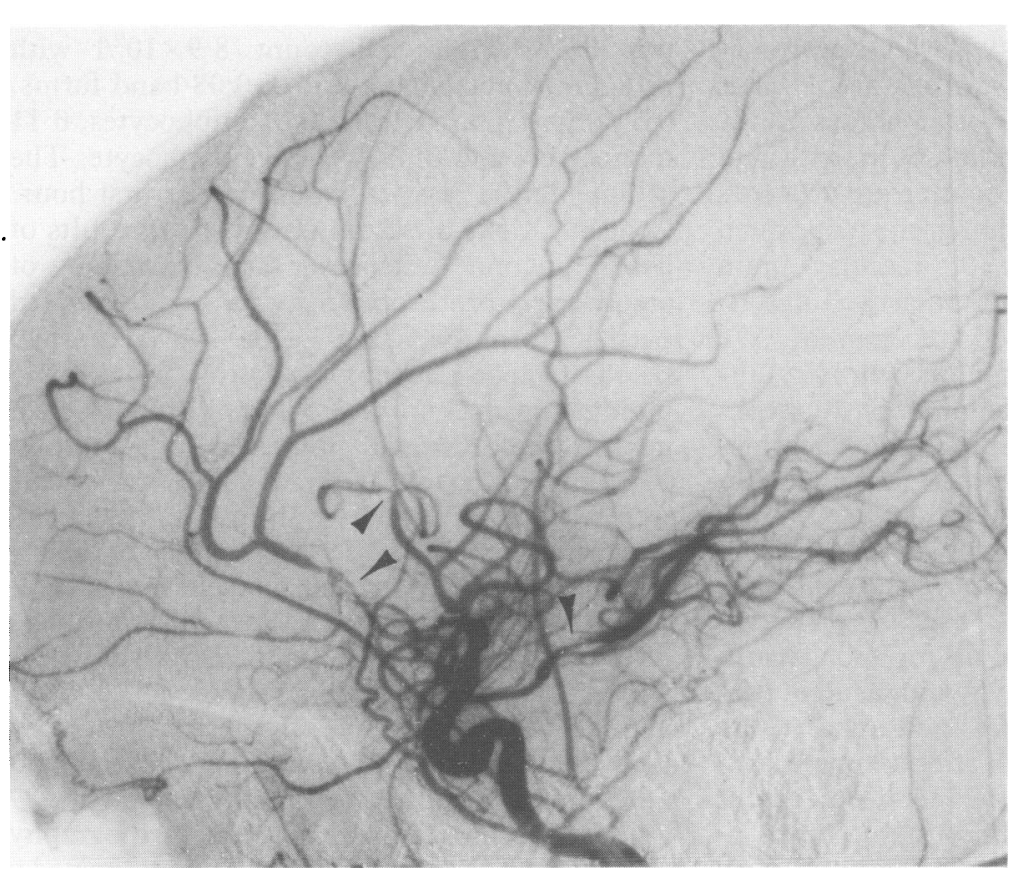

Figure 2 Cerebral angiography showing narrowing and beading of the left middle, anterior and, to a lesser degree, the posterior cerebral arteries. the arteries was considered to be typical of central nervous system vasculitis.

In view of the vasculitic appearance of the blood vessels, steroid treatment was initiated, first with intravenous methylprednisolone for three days and then oral prednisone for a period of two months. Over several days the child showed slow but progressive clinical improvement with some return of speech and improvement of his right hemiparesis. Seizure control was also noted to be excellent, leading to a $40 \%$ decrease in the valproic acid dose three months later. The blood concentration remained within the high therapeutic range.

Seven months after the acute stroke, the child presented again with acute onset of a flurry of myoclonic seizures and aphasia, and was admitted to the hospital. At this time his packed cell volume fraction was $\mathbf{0} \cdot 35$, white cell count $7 \cdot 2 \times 10^{9} / 1$, platelets $204 \times 10^{9} / 1$, and the sedimentation rate was $6 \mathrm{~mm} /$ hour. Concentrations of serum electrolytes, activities of liver enzymes, and anion gap and coagulation studies were normal. Blood lactate concentration was $1.6 \mathrm{mmol} / \mathrm{l}$. Amino acid patterns in serum and urine were normal. Rheumatoid factor, antiDNA antibodies and antinuclear antibodies were negative, and $\mathrm{C} 3$ and $\mathrm{C} 4$ concentrations were normal. Examination of the cerebrospinal fluid showed normal concentrations of glucose and protein, 2 mononuclear white blood cells $/ \mathrm{ml}$ and $121 \mathrm{red}$ blood cells $/ \mathrm{ml}$. The antivaricella immuofluorescent antibody titre was 1:1024 in the peripheral blood and 1:32 in the cerebrospinal fluid.

Electroencephalography showed bilateral multifocal polyspikes, and generalised slowing of the background, which was more pronounced on the left, where the basic rhythm was decreased at times to $1-3 / \mathrm{sec}$. The record suggested a diffuse encephalopathy. Magnetic

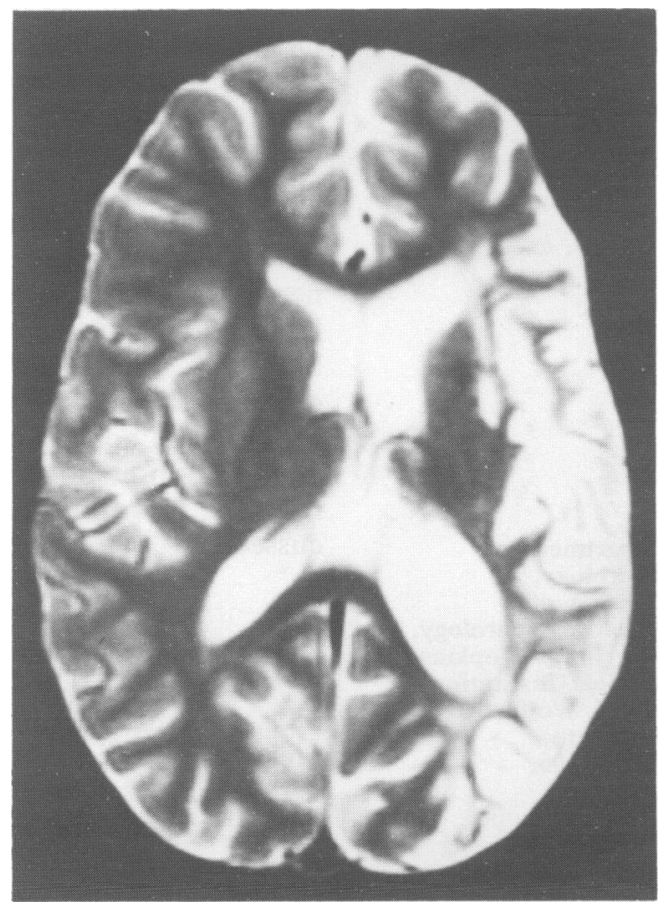

Figure 3 Magnetic resonance imaging of the brain showing silent posterior extension of the infarct. 
resonance imaging of the head showed an old posterior extension of the previous infarct (fig 3). Angiography was repeated, and showed noticeable resolution of the vasculitic process, except for a residual irregularity of the proximal portion of the left posterior cerebral artery.

The seizures were controlled by increasing the valproic acid to the previous dose and adding phenytoin. When seizures were controlled, neurologic examination showed that the child was in general back to his preadmission state, with recovery of the aphasia. Drooling, as well as some degree of increased mental dullness, persisted.

\section{Discussion}

This child presented with acute onset of right hemiparesis, with changes on magnetic resonance imaging compatible with acute cerebral infarction and angiographic evidence of unilateral central nervous system vasculitis. The lack of evidence for other causes of cerebral infarcts, such as heart disease, mitochondrial angiopathy, amino acid abnormalities, hypercoagulable state, or a collagen vascular disease, together with absence of systemic disease suggest the diagnosis of a primary central nervous system vasculitis. The angiographic pattern, with segmental narrowing and beading, is compatible with granulomatous angiitis of the nervous system, a rare disorder in the paediatric age group. ${ }^{2}$

The association between another clinical manifestation of varicella zoster virus-the herpes zoster ophthalmicus - and delayed hemiplegia is well documented. ${ }^{2}$ Within several weeks after the primary infection, patients with herpes zoster ophthalmicus can develop a contralateral hemiplegia, as a result of focal cerebral angiitis ipsilateral to the viral infection. Although the roentgenographic appearance of the affected vessels is similar to the cases of granulomatous angiitis of the nervous system, the two conditions differ clinically. In comparison with granulomatous angiitis of the nervous system, the herpes zoster ophthalmicus associated hemiplegia is generally less severe, is not associated with 'encephalopathic' symptoms, and is more limited in extent, and more likely to resolve. ${ }^{2}$

The mechanism for the vasculitis in herpes zoster ophthalmicus associated hemiplegia is unclear. Virus like particles have been reported in the cytoplasm and nuclei of smooth muscle cells from the wall of the affected vessel. ${ }^{2310} \mathrm{It}$ has been suggested that the virus is activated in the trigeminal ganglion and spreads to the adjacent carotid artery. Some patients with herpes zoster associated cerebral angiitis have had truncal rather than trigeminal zoster eruptions. In such patients, dissemination by either a haematogenous or cerebrospinal fluid pathway must be considered. ${ }^{11}$ Hilt et al demonstrated necrotising angiitis in a patient with herpes zoster ophthalmicus, but did not detect viral antigen in vessels. They suggested an immune mediated angiitis triggered by the herpes zoster as an alternate mechanism. Another patient with central nervous system vasculitis and underlying Hodgkin's disease was found to have antibodies to varicella zoster virus infection in the central nervous system without any previous vesicular eruption. ${ }^{5}$

The association of chickenpox and acute or delayed stroke is also documented. Griffith et al described a 31 year old woman who in the acute phase of chickenpox developed embolic occlusion of several cerebral arteries, due to sterile endocarditis. These authors have found more examples of chickenpox associated acute stroke in the literature. ${ }^{6}$ An additional case of a 26 year old patient who developed cerebral infarction 11 days into disseminated varicella was also reported. ${ }^{7}$ This patient showed evidence of mild systemic alteration in immune function, which was interpreted as suggestive of vasculitis. Kamholz and Tremblay described a 6 year old girl who developed a left hemiparesis six weeks after the onset of chickenpox. ${ }^{9}$ A computed tomogram showed hypodensity in the region of the right basal ganglia and small areas of enhancement in the right centrum semiovale. Arteriography showed stenosis of the proximal middle and anterior cerebral arteries with distal occlusions in both territories. The authors suggested that corneal inoculation could have resulted in contiguous spread of the virus to the internal carotid artery branches. Eda et al described four children (age range 1 to 5 years) with acute hemiplegia and lacunar infarcts that occurred one to three months after chickenpox. ${ }^{8}$ They failed, however, to demonstrate vasculitis in their patients.

The presumed association of varicella and vasculitis rests mainly on this temporal occurrence. The findings in the cerebrospinal fluid are not pathognomonic and may range from totally normal to a mild monocytic pleocytosis and a raised protein concentration. The presence of detectable varicella zoster antibodies in the cerebrospinal fluid (in a titre of $1: 2$ or greater) closely correlates with central nervous system infection with the virus, and is not found in infected patients without brain involvement. ${ }^{12}$ Nevertheless, this finding does not directly relate the vascular findings to the infection. Definite proof of this linkage can only be obtained by cerebral biopsy, which is not indicated in our patient.

Our patient's symptoms are similar to those of the five previously reported children, with the additional radiographic demonstration of unilateral angiitis resembling that reported in both granulomatous angiitis of the nervous system and after herpes zoster ophthalmicus. Antibody production within the central nervous system was suggested by a raised cerebrospinal fluid: serum antibody ratio $(1: 30)$. We speculate that during a process of generalised dissemination in the central nervous system, the virus was able to invade the wall of blood vessels, causing angiitis.

The subsequent course of our patient was not benign. Although the arteriography showed that the vasculitic process had improved, there was a silent extension of the infarct. Moreover, it may not be excluded that the presence of antibodies in the cerebrospinal fluid in an unchanged titre, represents a reactivation of the 
virus. Furthermore this reactivation could be the cause of the child's new onset of seizures and the evidence on electroencephalography of a diffuse encephalopathy.

The possible association between the delayed hemiplegia and chickenpox is clinically important. A large number of management related questions remain unanswered. If this disease entity carries a relatively good prognosis, steroids may not be needed. Indeed, because of a possible reactivation of the virus, steroids might be contraindicated and acyclovir would be indicated instead. The natural history of the vasculitis, and the possibility of recurrence of a thrombotic event, necessitating immunosuppressive or antiviral or antithrombotic treatment, are unknown.

It is possible that the acute vasculitis 10 weeks after chickenpox was an unfortunate coincidence. If so, it is an interesting coincidence. We suggest that, in the future, physicians treating acute strokes in children should carefully ask about a history of antecedent chickenpox, and proceed with an appropriate evaluation. The authors thank Dr Richard T Johnson, director of the departgist in chief, The Johns Hopkins Hospital, and Dr R Nick
Bryan, director of neuroradiology division, The Johns Hopkins Bryan, director of neuroradiology division, The Johns Hopkins
Hospital, for reviewing the manuscript and for their helpful comments.

AS is the recipient of a fellowship from the American Physicians Fellowship.

1 Kennedy PGE. Neurological complications of varicella-zoster virus. In: Kennedy PGE, Johnson RT, eds. Infections of the nervous system. London: Butterworths, 1987:177-208.

2 Sigal HL. The neurologic presentation of vasculitis and rheumatologic syndromes. Medicine 1987;66:157-80.

3 Morgello S, Block GA, Price RW, Petito CK. Varicella-zoster virus leukoencephalitis and cerebral vasculopathy. Arch Pathol Lab Med 1988;112:173-7.

4 Eidelberg D, Sotrel A, Horoupian S, Neuman PE, PumarolaSune T, Price RW. Thrombotic cerebral vasculopathy associated with herpes zoster. Ann Neurol 1986:19:7-14.

5 Vilchez-Padilla JJ, Redon J, Ruiz A, Lopez-Aldeguer J. CNS varicella-zoster vasculitis. Arch Neurol 1982;39:785.

6 Griffith JF, Salam MV, Adams RD. The nervous system diffith JF, Salam MV, Adams RD. The nervous system
diseases associated with varicella. Acta Neurol Scand 1970; diseases associa

7 Gibbs MA, Fisher M. Cerebral infarction in an adult with disseminated varicella. Bull Clin Neurosci 1986;51:65-7.

8 Eda I, Takashima S, Takeshia K. Acute hemiplegia with lacunar infarct after varicella infection in childhood. Brain Dev 1983;5:494-9.

9 Kamholz J, Tremblay G. Chickenpox with delayed contralateral hemiparesis caused by cerebral angitis. Ann Neurol 1985; 18:358-60.

10 Tanaka K, Hokamura K, Kinjo M. Fukumoto S. Subarachnoid hemorrhage and granulomatous angiitis of the basilar artery: demonstration of the varicella zoster virus in the basilar artery lesions. Stroke 1986;17:1024-8.

11 Hilt DC, Buchholz D, Krumholz A, Weiss H, Wolinsky JS. Herpes zoster ophthalmicus and delayed contralateral hemiparesis caused by cerebral angiitis: diagnosis and hemiparesis caused by cerebral angiitis: diagnosis and

12 Gershon A, Steinberg S, Greenberg S, Taber L. Varicellazoster associated encephalitis: detection of specific antibody in cerebrospinal fluid. $\mathcal{F}$ Clin Microbiol 1980;12:764-7. 